studies from no earliest date to December 2020 were included; adult patients with a severe and enduring mental illness prescribed antipsychotic medication were included. Cinician diagnosis, structured interview diagnosis, and interviewer or self-completion questionnaires were used to measure prevalence. The study designs included were experimental designs, cohort study, cross-sectional survey and administrative databases. Exclusion criteria being those with traumatic brain injury, psychosis secondary to autoimmune, iatrogenic, chromosomal or metabolic disorder, those with Learning disability or Autistic Spectrum disorders. studies with majority of participants $<18 \mathrm{yrs}$. Those who were on other antipsychotic medications in addition to Aripiprazole, were excluded. To ensure quality assurance, we used ROBINS-I tool and GRADE assessment to measure the risk of bias.

Result. 240 records were retrieved, 187 after duplicates were removed. 8 full text articles were assessed for eligibility, of which 4 were included in the qualitative synthesis. 2 studies were analyses of spontaneous adverse drug reaction reporting systems and 2 of health insurance claims databases. All 4 studies found aripiprazole to be associated with greater risk of impulse control disorders. The single study which compared directly with other antipsychotics had a much smaller effect size. Study heterogeneity precluded meta-analysis. All studies were at high risk of bias. The quality of evidence is very low.

Conclusion. The available evidence is consistent with the existing warnings regarding increased risk of impulse control disorders in patients prescribed aripiprazole. Clinicians may wish to monitor for this adverse drug reaction. Further research which can account for potential confounders, examines specific impulse control disorders and which is less susceptible to detection and ascertainment biases is required.

\section{Evaluating patients and healthcare professionals' understanding of voting rights for patients in government elections}

\author{
Mark Winchester*, Madiha Majid and Ashok Kumar \\ Coventry and Warwickshire Partnership Trust \\ ${ }^{*}$ Corresponding author.
}

doi: 10.1192/bjo.2021.796

Aims. To understand whether mental health patients vote in government elections

To ascertain the barriers that prevent them from doing so

To explore ways in which mental health services can support patients to vote

To determine whether mental health staff are aware of patients' right to vote

Background. Members of Parliament (MPs) can influence decisions regarding the National Health Service (NHS) and mental health legislation. The general election on 12th December 2019 highlighted that many patients were not using their democratic right to vote. It also appeared that many staff members were not aware that patients under the Mental Health Act (MHA) were entitled to vote (except for those under 'forensic' sections of the MHA). We therefore conducted a survey to ascertain both patient and staff understanding of their democratic rights and to better understand how we could increase the rate of voting amongst psychiatric patients.

Method. Two questionnaires were produced, one for patients and the other for staff members. This was tested by the clinical governance team before approval was granted. Data were collected at the Coventry and Warwickshire Partnership NHS Trust in the form of paper forms or electronically through a survey website. Forty-two patients and twenty-five staff members responded. Result. No staff members had received formal training with regards to patients' right to vote. Over half of staff members incorrectly believed that patients under Section 2 or 3 of the MHA and those lacking capacity couldn't vote. More than half of the team members surveyed stated that they had not supported patients in registering or casting a vote. Roughly one third of healthcare professionals felt that it was their responsibility to promote patients' right to vote, with one third disagreeing and the remaining third unsure.

Over $75 \%$ of patients did not vote but less than one quarter of all patients surveyed felt support from mental health services would increase the likelihood of them voting. The main barriers to voting were being mentally unwell, hospital admission or a lack of knowledge on the candidates and election process.

Conclusion. Basic training is required to improve staff knowledge of patients' voting rights, which should help improve their ability to support patients to vote. Trusts should have a clear protocol in place in the event of future elections, with information on who can vote, how to request a postal vote and the candidates in that area.

\section{A literature review of whether communication skills specific to psychiatry are being taught to medical undergraduates around the world}

Sarah Winfield ${ }^{1 \star}$ and Declan Hyland ${ }^{1}$

${ }^{1}$ Locum SHO, Windsor House, Liverpool, Mersey Care NHS

Foundation Trust and ${ }^{2}$ Consultant Psychiatrist, Clock View Hospital, Liverpool, Mersey Care NHS Foundation Trust

${ }^{*}$ Corresponding author.

doi: 10.1192/bjo.2021.797

Aims. The ability to communicate effectively is an imperative skill for clinicians to master as doctor-patient communication is one of the most essential dynamics in health care. Patients with a mental disorder present a unique challenge for doctors with regards to effective communication due to the nature of their illness.

This literature review aimed to determine whether medical undergraduates around the world are taught psychiatric communication skills.

Method. In January 2021, the following electronic databases were searched for articles relating to medical undergraduates, the concept of psychiatric communication skills and the teaching and support of such skill development: ERIC, MEDLINE, PsycINFO, SAGE and Web of Science. Combinations of keywords focussed the content of papers and truncation obtained alternative word endings. Generated articles were appraised iteratively for suitability against pre-defined inclusion criteria. The bibliographies of eligible articles were then examined to capture any further relevant studies. Ethical approval was not required.

Result. 1040 citations of potential relevance were initially identified. Following an iterative screening process, 10 articles (from seven different countries) were eligible for inclusion. $70 \%$ of papers used the modality of simulated patients to teach psychiatric communication skills and Technology Enhanced Learning (TEL) was used to create "virtual patients" for undergraduates to engage with. Discussing sensitive and emotive topics, such as suicide attempts or substance misuse, was less commonly taught compared to conditions such as anxiety and depression. Only $10 \%$ of papers explicitly taught medical undergraduates empathy or written communication skills and the importance placed on psychiatric teaching differed between countries. 
Conclusion. This literature review showed that some medical undergraduates receive psychiatric communication skills teaching, but the format and content of this varies. Increased consideration of incorporating TEL into psychiatric communications skills teaching is pertinent given undergraduates' reduced face-to-face patient contact during the COVID-19 pandemic, but further work is needed to validate such technology. Written communication skills are rarely taught but are imperative given the high volume of written correspondence in clinical practice. Delivering such teaching is feasible and should be incorporated into undergraduate curricula. Medical educators need to consider cultural differences when developing psychiatric communication skills teaching. Cultural influences not only affect undergraduate perceptions of psychiatry and mental illness, but also a patient's understanding and interpretation of their illness experience. Medical undergraduates may come from various cultural backgrounds, so actively discussing these differences opportunistically may augment the ability of medical undergraduates to be empathetic and establish therapeutic rapport with patients with mental illness.

\section{A study examining whether social cognitive abilities impact on recovery from PTSD}

Chantelle Wiseman ${ }^{1 \star}$, Jonathan Bisson ${ }^{2}$, Anke Karl ${ }^{3}$, Andrew Lawrence ${ }^{2}$, James Hotham ${ }^{4}$ and Stan Zammit ${ }^{5}$

${ }^{1}$ University of Bristol; ${ }^{2}$ Cardiff University; ${ }^{3}$ Exeter University; ${ }^{4}$ Avon and Wiltshire Partnership Trust and ${ }^{5}$ Cardiff University, Exeter

University

${ }^{\star}$ Corresponding author.

doi: 10.1192/bjo.2021.798

Aims. Deficits in social cognition (the ability to recognise and understand emotions, intentions and actions in oneself and in others) have been found in people with post-traumatic stress disorder (PTSD). Few studies so far have examined whether social cognitive ability impacts on PTSD recovery. Here we present a protocol and preliminary data for a study that aims to evaluate whether pre-treatment social cognitive deficits are associated with treatment outcomes following trauma-focused therapy for PTSD.

Method. The protocol was developed after discussion with Patient and Public Involvement (PPI) groups, and a battery of social cognitive tasks was trialled on 20 participants without PTSD. The final protocol was then developed using information and feedback from these preliminary studies. We aim to recruit 60 individuals who are about to start a trauma-focused therapy for PTSD within the two tertiary PTSD services. Social cognition (measured using a variety of tasks including Reading the Mind in the Eyes Task and the Reflective Functioning Questionnaire) and potential confounders (including severity of trauma history, attachment and verbal IQ) are assessed at baseline, prior to the start of therapy. PTSD symptom severity (measured using the PCL-5) and daily functioning (measured using the WSAS) are assessed pre and post-treatment. The primary aim of the study is to examine whether baseline social cognition is associated with the degree of improvement in the PCL-5.

Result. So far 29 participants have been recruited and consented. 6 participants have completed follow-up assessments. The study has been adapted for the COVID-19 pandemic so participants can complete the tasks remotely. Preliminary results show a moderate negative correlation between baseline social cognitive abilities and baseline PTSD symptom severity (Spearman's correlation -0.30) and functional abilities (Spearman's correlation -0.42).
Conclusion. Development of our study in collaboration with PPI and preliminary testing of measures suggests it is likely that it will be feasible for us to conduct this study in this patient group. Baseline preliminary results show/suggest a moderate correlation between PTSD symptom severity and social cognitive impairment. Our main analyses, when completed, will help to determine whether social cognitive ability is associated with recovery from PTSD.

\section{Exploring evidence of fatigue in survivors of paediatric brain tumours: a systematic review}

Jennifer Wood ${ }^{1 \star}$ and Sarah Verity ${ }^{2}$

${ }^{1}$ Newcastle University Medical school and ${ }^{2}$ Newcastle upon Tyne Hospitals NHS FT, Newcastle University Centre for Cancer ${ }^{*}$ Corresponding author.

doi: 10.1192/bjo.2021.799

Aims. As the number of survivors of childhood brain tumor grows, fatigue is being increasingly recorded as a long-term consequence of both the cancer itself and the treatment received. Survivors of childhood brain tumour report more significant fatigue than children with other cancers, often impacting all aspects of life, including academic attainment, self-concept and social relationships with peers, leading to reduced health-related quality of life.

This study aimed to systematically evaluate the evidence for fatigue in paediatric brain tumour survivors.

Method. A systematic search using EMBASE, MEDLINE and PsycINFO identified 20 papers meeting the inclusion criteria. Scientific rigor was used throughout by following Scottish Intercollegiate Guidelines Network (2015) guidance for systematic reviews. Quality Assessment of Evidence Rating tool - Fatigue (QAERT) was developed with substantial inter-rater agreement found.

Result. 19 of the 20 studies reviewed showed conclusive evidence of fatigue in survivors of paediatric brain tumour. One study offered adequate evidence that there was no difference in levels of fatigue in paediatric cancer survivors, including survivors of paediatric brain tumour, when compared to healthy controls. Three studies found that fatigue was worse in survivors of paediatric brain tumour when compared to survivors of other paediatric cancers

Conclusion. This review provides evidence for the presence of fatigue in survivors of paediatric brain tumour. However, the construct of fatigue was poorly defined throughout, with fatigue associated with physical effects of treatment and fatigue associated with long-term cognitive impairment not distinguished. This poor construct validity, coupled with a lack of comparison groups in 12 of the 20 studies, reduces the generalizability of the data and its usefulness for developing effective psychological interventions. Further research is needed, built on a clear fatigue construct definition, and including well defined exclusion criteria, to provide a sound basis for improving the quality of life of these children.

Pre-discharge factors associated with early readmission to psychiatric inpatient services within 90 days

Jessica Wright* and Rhianne Thomas

Barnet, Enfield and Haringey NHS Mental Health Trust ${ }^{\star}$ Corresponding author.

doi: 10.1192/bjo.2021.800 